Claremont Colleges

Scholarship@ Claremont

Pomona Faculty Publications and Research

Pomona Faculty Scholarship

$1-1-1986$

\title{
A Prime Strongly Positive Amphicheiral Knot Which Is Not Slice
}

Erica Flapan

Pomona College

\section{Recommended Citation}

Erica Flapan (1986). A prime strongly positive amphicheiral knot which is not slice. Mathematical Proceedings of the Cambridge Philosophical Society, 100, pp 533-537. doi:10.1017/S0305004100066263.

This Article is brought to you for free and open access by the Pomona Faculty Scholarship at Scholarship @ Claremont. It has been accepted for inclusion in Pomona Faculty Publications and Research by an authorized administrator of Scholarship @ Claremont. For more information, please contact scholarship@cuc.claremont.edu. 


\section{Mathematical Proceedings of the Cambridge Philosophical Society}

http://journals.cambridge.org/PSP

Additional services for Mathematical Proceedings of the Cambridge Philosophical Society:

Email alerts: Click here

Subscriptions: Click here

Commercial reprints: Click here

Terms of use : $\underline{\text { Click here }}$

MATHEMATICAL

PROCEEDINGS

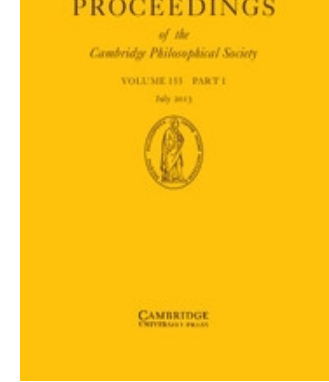

\section{A prime strongly positive amphicheiral knot which is not slice}

\section{Erica Flapan}

Mathematical Proceedings of the Cambridge Philosophical Society / Volume 100 / Issue 03 / November 1986, pp $533-537$

DOI: 10.1017/S0305004100066263, Published online: 24 October 2008

Link to this article: http://journals.cambridge.org/abstract S0305004100066263

How to cite this article:

Erica Flapan (1986). A prime strongly positive amphicheiral knot which is not slice. Mathematical Proceedings of the Cambridge Philosophical Society, 100, pp 533-537 doi:10.1017/ S0305004100066263

Request Permissions : $\underline{\text { Click here }}$ 


\title{
A prime strongly positive amphicheiral knot which is not slice
}

\author{
BY ERICA FLAPAN \\ Department of Mathematics, University of California, \\ Santa Barbara, CA 93106, U.S.A.
}

(Received December 1985; revised 25 February 1986)

We begin by giving several definitions. A knot $K$ in $S^{\mathbf{3}}$ is said to be amphicheiral if there is an orientation-reversing diffeomorphism $h$ of $S^{3}$ which leaves $K$ setwise invariant. Suppose, in addition, that $K$ is given an orientation. Then $K$ is said to be positive amphicheiral if $h$ preserves the orientation of $K$. If, in addition, the diffeomorphism $h$ is an involution then $K$ is strongly positive amphicheiral. Finally, we say a knot is slice if it bounds a smooth disc in $B^{4}$. In this note we shall give a smooth example of a prime strongly positive amphicheiral knot which is not slice.

Levine [5] introduced an algebraic condition which is necessary for a knot to be slice. A knot satisfying this condition is said to be 'algebraically slice'. Recently, D. Long [8] showed that all strongly positive amphicheiral knots are algebraically slice. Long concludes his paper with the question of whether all prime strongly positive amphicheiral knots are, in fact, slice. So, our example answers Long's question in the negative.

To build our example we start with a knot $L$ which is not concordant to its reverse (the 'reverse' of a knot is the knot with its string orientation reversed). We choose $L$ to be the simplest of the collection of such knots constructed by Livingston [7]. Let $L^{\prime}$ denote the connected sum of our $\operatorname{knot} L$ with its mirror image $L^{*}$. Since $L$ is not concordant to its reverse, the knot $L^{\prime}$ is not slice. Fig. 1 illustrates the knot $L$, and Fig. 2 illustrates this connected sum $L^{\prime}$.

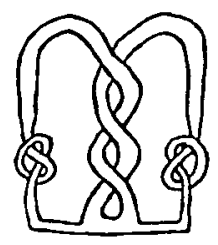

Fig. 1. $L$.

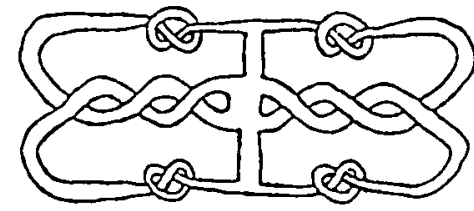

Fig. 2. $L^{\prime}$.

Let $h$ be the diffeomorphism of $S^{3}$ which is given by first reflecting through the plane of the page containing the projection of $L^{\prime}$, then rotating by $\pi$ about an axis perpendicular to the page through the centre of the projection. This diffeomorphism is an orientation-reversing involution which preserves the orientation of $L^{\prime}$. Thus $L^{\prime}$ is an example of a composite knot which is strongly positive amphicheiral but is not slice. We will now show that the knot $K$, which is shown in Fig. 3, is a prime such example.

Our example $K$ is concordant to $L^{\prime}$ and hence also is not slice. This is because performing band moves along bands parallel to the top of each of the clasps would again yield the $\operatorname{knot} L^{\prime}$. The fact that $K$ is strongly positive amphicheiral is clear by observing that again a reflection through the plane of the paper followed by a rotation 


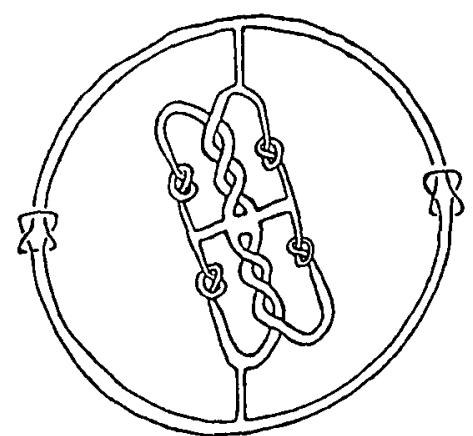

Fig. 3. $K=$ a prime strongly positive amphicheiral knot which is not slice.

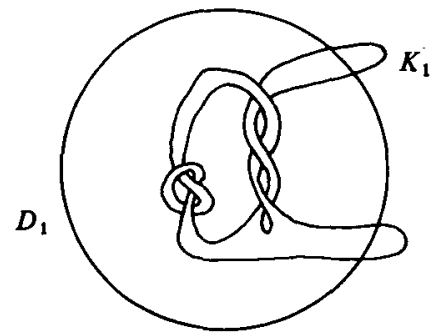

Fig. $4 a .\left(D_{1}, D_{1} \cap K_{1}\right)$.

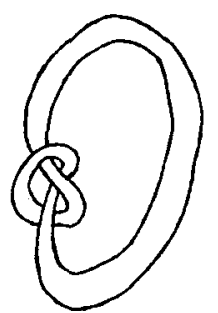

Fig. 4 b. $K_{1}$.

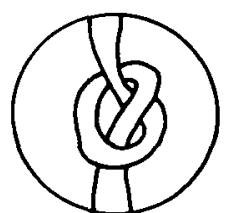

Fig. 5. $\left(D_{2}, D_{2} \cap K_{2}\right)$.

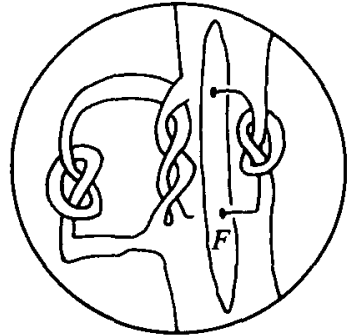

Fig. 6. $(A, A \cap L)$.

by $\pi$ about a central axis provides the desired involution. We shall show that $K$ is prime by reconstructing $K$ out of prime tangles and using the methods of Lickorish [6].

We start with the link $K_{1}$ and the tangle $\left(D_{1}, D_{1} \cap K_{1}\right)$ which are both defined by Fig. $4 a$. Fig. $4 b$ shows $K_{1}$ after an isotopy.

It can be easily checked that $K_{1}$ is a prime link. So, by definition, any 2 -sphere that meets $K_{1}$ transversely in two points, bounds a ball in $S^{3}$ which intersects $K_{1}$ in a single unknotted spanning arc. This observation allows us to show that $\left(D_{1}, D_{1} \cap K_{1}\right)$ is a prime tangle. First, note that this tangle is not the untangle since each of the ares of $D_{1} \cap K_{1}$ is a trefoil knot. So, if $\left(D_{1}, D_{1} \cap K_{1}\right)$ is not prime, then there is a 2-sphere in $D_{1}$ which meets $K_{1}$ transversely in two points and bounds a ball $E$ meeting $D_{1} \cap K_{1}$ in a knotted arc. Since $K_{1}$ has two components, the complementary ball $S^{3}-E$ meets $K_{1}$ in an arc together with one complete component of $K_{1}$. Thus neither ball intersects $K_{1}$ in a single unknotted spanning arc. However, this contradicts the primeness of the link $K_{1}$. Hence, we conclude that the tangle $\left(D_{1}, D_{1} \cap K_{1}\right)$ was, in fact, prime. 
Let $\left(D_{2}, D_{2} \cap K_{2}\right)$ be the double trefoil tangle which is illustrated in Fig. 5. This tangle is easily shown to be prime (see Lickorish [6], example $2 b$, or use the same argument as above).

Recall that $L$ was the Livingston knot shown in Fig. 1. Let Fig. 6 define the tangle $(A, A \cap L)$, together with a properly embedded disc $F$ which separates it into the two prime tangles $\left(D_{1}, D_{1} \cap K_{1}\right)$ and $\left(D_{2}, D_{2} \cap K_{2}\right)$. Thus, it follows from theorem 3 of [6] that the tangle $(A, A \cap L)$ is prime.

We shall let the tangle $\left(A^{*}, A^{*} \cap L^{*}\right)$ denote the mirror image of the tangle $(A, A \cap L)$. Then it is clear that $\left(A^{*}, A^{*} \cap L^{*}\right)$ is also a prime tangle. So, again by theorem 3 of [6], we conclude that the tangle $\left(B, B \cap L^{\prime}\right)$, of Fig. 7, is prime. Furthermore, as Kirby and Lickorish [3] noted, the clasp tangle $(C, C \cap J)$, of Fig. 7, is prime as well.
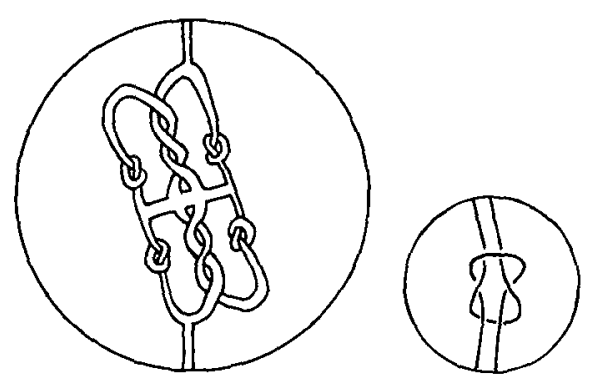

Fig. 7. $\left(B, B \cap L^{\prime}\right) \quad(C, C \cap J)$.

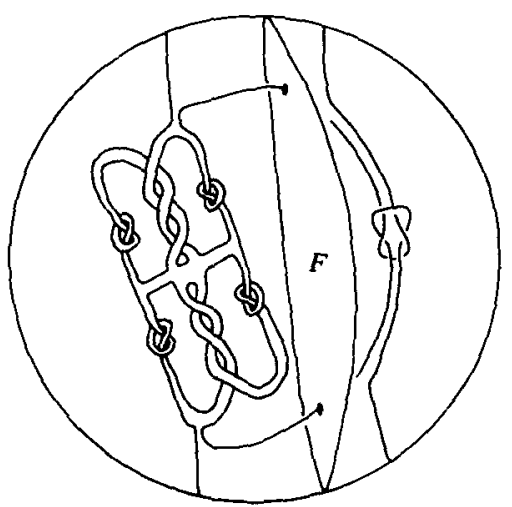

Fig. 8, $\left(D, D \cap L^{\prime \prime}\right)$ together with the dise $F$.

Figure 8 illustrates the tangle $\left(D, D \cap L^{\prime \prime}\right)$ together with a properly embedded disc $F$ which separates $\left(D, D \cap L^{\prime \prime}\right)$ into the two prime tangles $\left(B, B \cap L^{\prime}\right)$ and $(C, C \cap J)$. So, once again we apply theorem 3 of [6] to see that the tangle $\left(D, D \cap L^{\prime \prime}\right)$ is prime.

Finally, our example $K$ is the sum of the tangle $\left(D, D \cap L^{\prime \prime}\right)$ with another copy of the clasp tangle $(C, C \cap J)$. So, by theorem 1 of [6], the knot $K$ is prime.

Using a similar method we can also answer a question of Hartley and Kawauchi [2]. In [2], they show that the Alexander polynomial $A(t)$ of any strongly positive amphicheiral knot is a square, $A(t)=f(t)^{2}$. Conversely, if $f(t)$ is the Alexander polynomial of a knot $L$, then the $\operatorname{knot} L^{\prime}$, which is the connected sum of $L$ with its mirror image $L^{*}$, is a strongly positive amphicheiral knot with Alexander polynomial $f(t)^{2}$. Hartley and Kawauchi ask the question of whether for a given $f(t)$ there is a prime knot which is

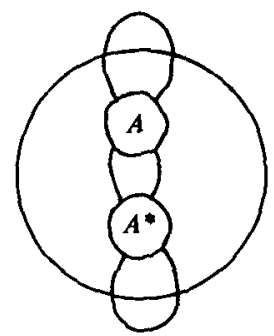

Fig. 9. $\left(B, B \cap L^{\prime}\right)$ 
strongly positive amphicheiral and has Alexander polynomial $f(t)^{2}$. We answer this question in the affirmative as follows.

Given the knot $L$, by theorem 4 of [6], we can write $L$ as the sum of a prime tangle $(A, A \cap L)$ together with the untangle. Also, the mirror image tangle $\left(A^{*}, A^{*} \cap L^{*}\right)$ is prime. So again, as in our example above, by theorem 3 of [6] the tangle $\left(B, B \cap L^{\prime}\right)$ shown in Fig. 9, is a prime tangle.

Now let $J$ be the Kinoshita-Terasaka knot [4]; then it follows from Bleiler [1] that the tangle $(C, C \cap J)$, shown in Fig. 10, is also a prime tangle.
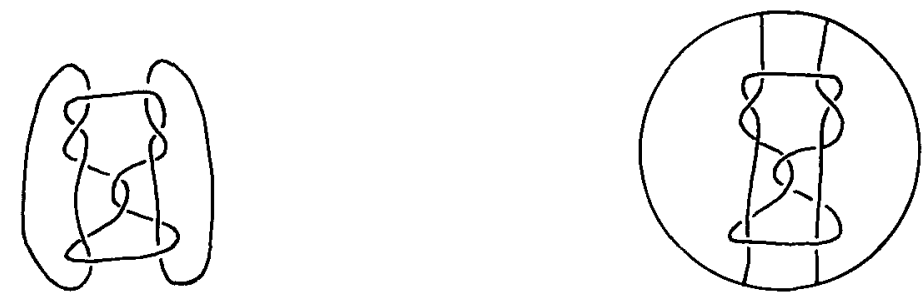

Fig. 10. $J=$ the Kinoshita-Terasaka knot $(C, C \cap J)$.

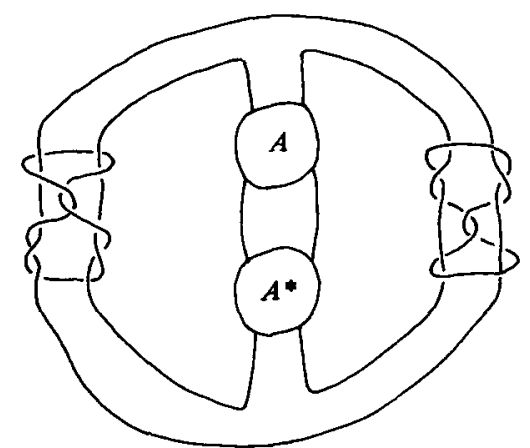

Fig. 11. $K=$ a prime strongly positive amphicheiral knot with Alexander polynomial $A(t)=f(t)^{2}$.

Here the desired knot $K$ is obtained by taking the sum of the tangle $\left(B, B \cap L^{\prime}\right)$ with one copy of the tangle $(C, C \cap J)$ and one copy of its mirror image, as indicated in Fig. 11.

The knot $K$ is prime and strongly positive amphicheiral for the same reasons as our first example. By using the fact that the Kinoshita-Terasaka knot has trivial Alexander polynomial, Bleiler [1] has shown that adding the Kinoshita-Terasaka tangle to a given knot has no effect on its Alexander polynomial. Thus $K$ is, in fact, a prime strongly positive amphicheiral knot which has Alexander polynomial $f(t)^{2}$.

The author was partially supported by a grant from the O.N.R.

\section{REFERENCES}

[1] S. BLeILer. Realizing concordant polynomials with prime knots. Pacific J. Math. 100 (1982), 249-257.

[2] R. Hartuey and A. Kawauchi. Polynomials of amphicheiral knots. Math. Ann. 243 (1979) 63-70.

[3] R. Kirby and W. B. R. Lickorish. Prime knots and concor dance. Math. Proc. Cambridge Philos. Soc. 86 (1979), 437-441.

[4] S. Kinoshita and H. Terasaka. On unions of knots. Osaka Math. J. 9 (1959), 131-153. 
[5] J. Levine. Knot cobordism groups in codimension two. Comment. Math. Helv. 44 (1969), 229-244.

[6] W. B. R. Lickonish. Prime knots and tangles. Trans. Amer. Math. Soc. 267 (1981), 321332.

[7] C. Livingston. Knots which are not concordant to their reverses. Quart. J. Math. Oxford 34 (1983), 323-328.

[8] D. D. Lova. Strongly plus-amphicheiral knots are algebraically slice. Math. Proc. Cambridge Philos. Soc. 95 (1984), 309-311. 